\title{
A HYBRID PROCEDURE TO IDENTIFY THE OPTIMAL STIFFNESS COEFFICIENTS OF ELASTICALLY RESTRAINED BEAMS
}

\author{
Tiago SILVA ${ }^{a, b, *}$, MARIA LOJA $^{a, b}$, NUNO MAIA $^{b}, \operatorname{JOAQUim~BARBOSA~}^{a, b}$ \\ ${ }^{a}$ Research Group on Modelling and Optimization of Multifunctional Systems (GI-MOSM) \\ Mechanical Engineering Department, Instituto Superior de Engenharia de Lisboa (ADEM/ISEL) \\ Rua Conselheiro Emídio Navarro, 1, 1959-007 Lisboa, Portugal \\ e-mail: tasilva@dem.isel.pt
}

${ }^{b}$ LAETA, IDMEC, Instituto Superior Técnico, Universidade de Lisboa Avenida Rovisco Pais, 1, 1049-001 Lisboa, Portugal

\begin{abstract}
The formulation of a bending vibration problem of an elastically restrained Bernoulli-Euler beam carrying a finite number of concentrated elements along its length is presented. In this study, the authors exploit the application of the differential evolution optimization technique to identify the torsional stiffness properties of the elastic supports of a Bernoulli-Euler beam. This hybrid strategy allows the determination of the natural frequencies and mode shapes of continuous beams, taking into account the effect of attached concentrated masses and rotational inertias, followed by a reconciliation step between the theoretical model results and the experimental ones. The proposed optimal identification of the elastic support parameters is computationally demanding if the exact eigenproblem solving is considered. Hence, the use of a Gaussian process regression as a meta-model is addressed. An experimental application is used in order to assess the accuracy of the estimated parameters throughout the comparison of the experimentally obtained natural frequency, from impact tests, and the correspondent computed eigenfrequency.
\end{abstract}

Keywords: transverse vibration, Bernoulli-Euler beam, elastic support, torsional stiffness coefficient, differential evolution, Kriging predictor.

\section{Introduction}

The study of beam-like components that have cross section variations along the length and/or carry concentrated masses and/or springs is often addressed by means of approximated numerical methods, such as Rayleigh's quotient. The accuracy of such an approach depends on the chosen shape function, according to Rayleigh's theorem (Meirovitch, 2001). The objective of the present work is to develop an accurate model for generic physical systems, as illustrated in Fig. 1, in order to replicate the experimental natural frequencies in lateral bending. Thus, an automated procedure to establish and to solve the symbolic eigenproblem, derived from classical beam theory, is proposed.

While the rotor itself presents no problem and can easily be studied as a Bernoulli-Euler beam with a concentrated mass, the identification of the adequate

* Corresponding author linear and torsional stiffness properties associated with the end supports remains a challenge. Note that the term 'torsional' is related to a constraint of the angular displacement in the $x-z$ plane (Fig. 1). In a similar context, De Rosa et al. (1996) supposed the beam to be elastically restrained against rotation and translation at both ends, so that it was possible to study all the common boundary conditions. They showed that trigonometric functions work slightly better than the static deflections and highlighted the accuracy of Rayleigh's quotient to the true frequencies. Several authors present exact solutions for the frequency equation of a Bernoulli-Euler beam restricting the stiffness coefficients, in order to reproduce some particular cases, and accounting for the rotation inertia of attached discs and their eccentricity (De Rosa and Auciello, 1996; Auciello, 1996; Grossi and Albarracín, 2003; Maiz et al., 2007). Similar problems are treated by Nallim and Grossi (1999) as well as Albarracín 


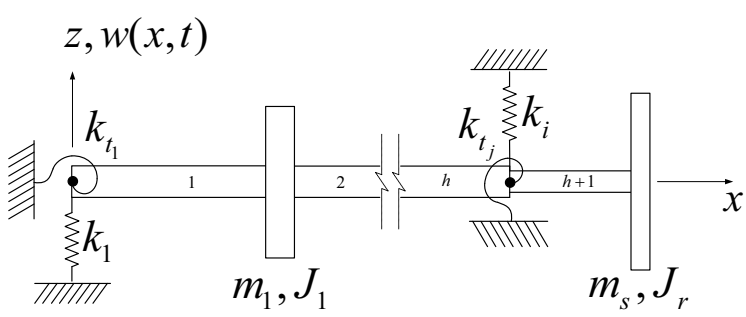

Fig. 1. Generic beam model with $h$ intermediate discontinuities.

et al. (2004), who consider intermediate supports.

Wu and Chen (2003) studied the bending vibrations of wedge Bernoulli-Euler beams with any number of point masses, verifying that the mass distribution on the beam affects the dynamical behaviour more than the mass addition itself. The works of Biondi and Caddemi $(2005 ; 2007)$ treat beams with discontinuities in the curvature or in the slope functions, allowing the adoption of different materials or different cross sections in the model. The formulation for multi-stepped discontinuities on Bernoulli-Euler beams is presented by Bashash et al. (2008) and Vaz (2008), the latter giving a useful explanation for the computational implementation for the bending vibration problem of beams with a infinite number of geometrical discontinuities. Elishakoff and Pentaras (2006) worked on the free vibration of non-homogeneous Bernoulli-Euler beams using a polynomial approach for the mode shape of the natural frequency of interest. The developed formulation does not require numerical modelling and provides a useful tool to the design phase. The above referred papers deal with slender beams, typically those with a length-to-diameter ratio greater than $10, l / D>10$. As a consequence, the inertial rotation energy of an infinitesimal element $\mathrm{d} x$ is negligible compared with its translational energy, justifying the applicability of the Bernoulli-Euler beam theory. Otherwise, the problem has to be treated using the Timoshenko beam theory (Posiadała, 1997; Lin and Hsiao, 2001).

The identification of structural boundary conditions is a concern, as the lower frequency dynamical response of a structure is often dominated by these conditions. Hence, several authors worked on different approaches in order to identify the parameters of generic elastic supports. Wang and Yang (2011) established a direct method to identify the stiffness parameters of tapered Bernoulli-Euler beams with an elastically supported end. The identification process is based on measured static flexibility functions throughout the application of a weighted least squares method. As the boundary conditions imposed by elastic supports depend on the spatial derivatives of the displacement, Chesne (2012) proposed to identify the stiffness parameters in Bernoulli-Euler beams elastically supported at both ends and excited by harmonic loads, through the estimation of the referred derivatives. The author approximates the shape function by a truncated Taylor series, from which the estimated spatial derivatives are extracted using annihilators, as described in the work of Mboup et al. (2009). Majkut (2006) approached the same problem, although the use of singular value decomposition is addressed in order to compute the unknown stiffness parameters in a least squares sense. Modeling a structure by finite elements is an alternative to its analytical formulation, where the effect of the supports can be introduced by adding a set of nodal forces at the boundary nodes. Additionally, the relation between nodal displacements and nodal forces is given by the dynamic stiffness matrix. Thus, Ahmadian et al. (2001) proposed to identify the structural boundary conditions through the reconciliation of the measured and predicted modal responses, eliminating the unknown nodal reaction forces by the introduction of the support stiffness matrix. Similarly, Yoneyama and Arikawa (2012) proposed a boundary condition identification method based on full-field measured displacements.

In the present work, we shall use Rayleigh's quotient, with exact shape functions that take into account the flexibility of the supports and the effect of the mass and torsional inertia of attached discs, in order to identify the value of the torsional stiffness of both supports as proposed by Silva and Maia $(2011 ; 2013)$. It is worth mentioning that the computation of the exact shape functions is fully automated and therefore not limited to the presented experimental example (Figs. 2 and 3). In order to identify the referred stiffness parameters, the differential evolution (DE) algorithm is used to find the optimal stiffness parameters, i.e., the ones that minimize the relative difference between experimental and analytical eigenfrequencies. Due to the demanding computational effort of evaluating the eigenproblem hundreds of times, we shall address the use

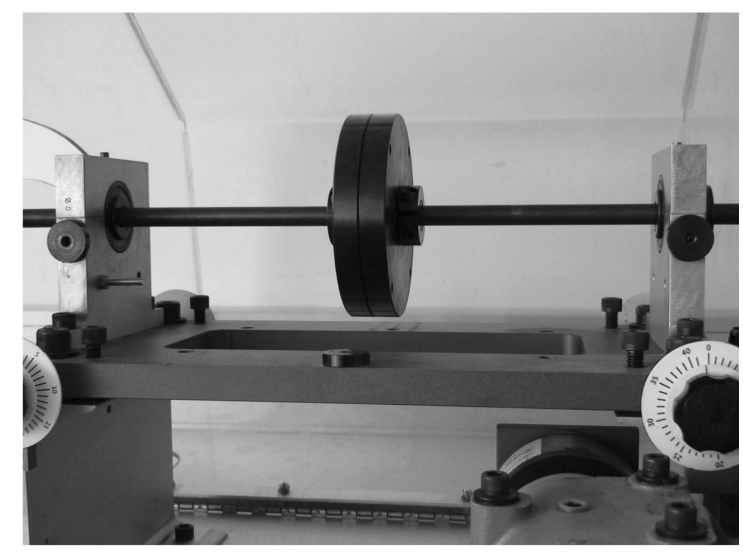

Fig. 2. System under study (MFS, SpectraQuest, Inc.). 
of a Kriging predictor as a meta-model, built from several training evaluations of the eigenproblem. In the following sections, one can find the theoretical background on the bending vibrations of Bernoulli-Euler beams on elastic supports and an experimental example where the accuracy of the identified optimal solutions is assessed.

\section{Theoretical background}

To obtain an analytical model that reproduces a generic physical system, we consider Rayleigh's quotient, using the Bernoulli-Euler beam theory to define the shape functions. In previous works of Silva and Maia (2011; 2013), it was shown that the simplification introduced by the ideal cases, simply supported and clamped beam ends, leads to results that are significantly different from the experimental ones. Therefore, it is proposed to build a model (Fig. 1) where a Bernoulli-Euler beam can be elastically restrained and present any kind of intermediate discontinuities, without taking any specific assumption.

Regarding Rayleigh's method, the energy dissipation is neglected and therefore the principle of conservation of energy holds:

$$
\Delta(T+V)=0 \Rightarrow T_{\max }=V_{\max },
$$

where $T$ and $V$ are the kinetic and potential energies, respectively.

Through the quantification of the work performed by the elastic forces, in relation to the equilibrium position of the beam, the potential energy is

$$
V=\frac{1}{2} \int_{0}^{l} M(x, t) \mathrm{d} \theta,
$$

where $M(x, t)$ is the bending moment given by $M(x, t)=\partial^{2}(E(x) I(x) w(x, t)) / \partial x^{2}, l$ is the length of the beam and $\theta$ is the rotation angle given by $\theta(x, t)=$ $\partial w(x, t) / \partial x . E(x)$ is the Young modulus and $I(x)$ is the second moment of the area of the beam, while $w(x, t)$ represents the lateral displacement of the beam in relation to its equilibrium position.

At each undamped natural frequency, the time variation of $w(x, t)$ can be shown to be harmonic and

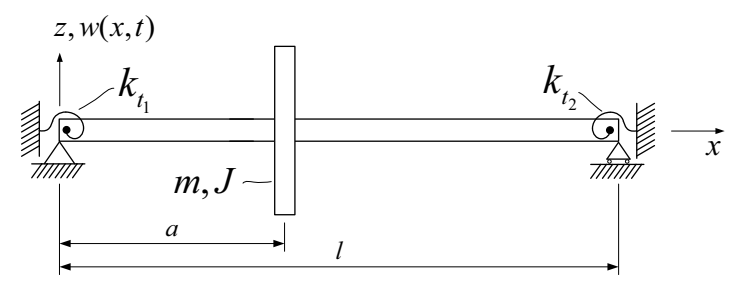

Fig. 3. Model of a beam with elastic torsional supports and a rigid disc at $x=a$. therefore the displacement response is given by the harmonic variation of a shape function $\phi(x)$,

$$
w(x, t)=\phi(x) \sin (\omega t+\varphi) .
$$

From Eqn. (3), $w(x, t)_{\max }=\phi(x)$, leading to

$$
V_{\text {max }}=\frac{1}{2} \int_{0}^{l} E(x) I(x)\left(\phi^{\prime \prime}(x)\right)^{2} \mathrm{~d} x .
$$

On the other hand, the kinetic energy is given by

$$
T=\frac{1}{2} \int_{0}^{l} \dot{w}(x, t)^{2} \mathrm{~d} m .
$$

From Eqn. (3), $\dot{w}(x, t)_{\max }=\omega \phi(x) . \quad$ As $\mathrm{d} m=$ $\rho(x) A(x) \mathrm{d} x$, where $\rho(x)$ is the density of the material and $A(x)$ the cross sectional area of the beam, we have

$$
T_{\max }=\omega^{2} \frac{1}{2} \int_{0}^{l} \rho(x) A(x)(\phi(x))^{2} \mathrm{~d} x .
$$

2.1. Rayleigh's quotient. Rayleigh's quotient $R$ results from the application of Eqn. (1) and, therefore, for a beam with no extra masses and springs, Eqns. (4) and (6) lead to

$$
R=\omega^{2}=\frac{\int_{0}^{l} E(x) I(x)\left(\phi^{\prime \prime}(x)\right)^{2} \mathrm{~d} x}{\int_{0}^{l} \rho(x) A(x)(\phi(x))^{2} \mathrm{~d} x},
$$

where the prime denotes differentiation with respect to the spatial coordinate $x$.

Rayleigh's quotient has several interesting properties from the numerical point of view. One of them is the upper bound approximation of the natural frequency value, as long as we provide a shape function $\phi(x)$ close enough to a true mode shape, respecting at least the geometric boundary conditions of the problem. As a first order variation on $\phi(x)$ corresponds to a second order variation on $\omega^{2}$, Rayleigh's quotient has a stationary value (a minimum in this case) in the neighbourhood of the true natural frequency. This is satisfied not only for the fundamental natural frequency, but for all of them, i.e., an accurate approximation for the $n$-th shape function $\phi_{n}(x)$ leads to an accurate approximation for the $n$-th natural frequency $\omega_{n}^{2}$.

Taking into account the possible existence of concentrated parameters along the length of the beam, such as stiffness coefficients and masses and corresponding inertias, we can rewrite Eqns. (4) and (6). Therefore, the total maximum value for the potential energy $V_{\max }$ will be given by

$$
V_{\max }=V_{b}+\sum_{i} V_{k_{i}}+\sum_{j} V_{k_{t_{j}}}
$$

where $V_{b}$ is the potential energy of the beam itself, given by Eqn. (4), $V_{k_{i}}$ is the potential energy of each local 
translational stiffness $k_{i}$, and $V_{k_{t_{j}}}$ is the potential energy of each local torsional stiffness $k_{t_{j}}$, respectively located at $x=x_{i}$ and $x=x_{j}$,

$$
\begin{aligned}
V_{k_{i}} & =\left.\frac{1}{2} k_{i} \phi_{n}(x)\right|^{2}, \\
V_{k_{t_{j}}} & =\left.\frac{1}{2} k_{t_{j}} \phi_{n}^{\prime}(x)\right|_{x=x_{j}} ^{2} .
\end{aligned}
$$

The maximum kinetic energy $T_{\max }$ will be given by

$$
T_{\max }=T_{b}+\sum_{r} T_{m_{r}}+\sum_{s} T_{J_{s}},
$$

where $T_{b}$ is the kinetic energy of the beam itself, given by Eqn. (6), $T_{m_{r}}$ is the kinetic energy of each local mass $m_{r}$, and $T_{J_{s}}$ is the kinetic energy of each local inertia $J_{s}$, respectively located at $x=x_{r}$ and $x=x_{s}$,

$$
\begin{aligned}
T_{m_{r}} & =\left.\frac{1}{2} \omega^{2} m_{r} \phi_{n}(x)\right|_{x=x_{r}} ^{2}, \\
T_{J_{s}} & =\left.\frac{1}{2} \omega^{2} J_{s} \phi_{n}^{\prime}(x)\right|_{x=x_{s}} ^{2} .
\end{aligned}
$$

Considering Eqns. (8) and (10), recalling Eqn. (1), the general expression of Rayleigh's quotient is defined as

$$
\begin{aligned}
R_{n}= & \omega_{n}^{2} \\
= & {\left[\int_{0}^{l} E(x) I(x)\left(\phi_{n}^{\prime \prime}(x)\right)^{2} \mathrm{~d} x\right.} \\
& \left.+\left.\sum_{i} k_{i} \phi_{n}(x)\right|_{x=x_{i}} ^{2}+\left.\sum_{j} k_{t_{j}} \phi_{n}^{\prime}(x)\right|_{x=x_{j}} ^{2}\right] \\
& \times\left[\int_{0}^{l} \rho(x) A(x)\left(\phi_{n}(x)\right)^{2} \mathrm{~d} x\right. \\
& \left.+\left.\sum_{r} m_{r} \phi_{n}(x)\right|_{x=x_{r}} ^{2}+\left.\sum_{s} J_{s} \phi_{n}^{\prime}(x)\right|_{x=x_{s}} ^{2}\right]^{-1} .
\end{aligned}
$$

2.2. Shape functions. The selection of the shape function may have a significant impact on the eigenvalue approximated by Rayleigh's quotient. The point is how to choose such an appropriate shape function, which should at least verify the geometric boundary conditions, although more accurate results are expected if it satisfies the natural boundary conditions, too. Moreover, it is also known that, in general, trigonometric functions provide better results than polynomial ones or those based upon static deflections. A possible choice which gives very good results in many cases would be to take the shape function that results from the solution of the equilibrium equation of the Bernoulli-Euler beam theory for free vibrations,

$$
c^{2} \frac{\partial^{4} w(x, t)}{\partial x^{4}}+\frac{\partial^{2} w(x, t)}{\partial t^{2}}=0
$$

where $c^{2}=E I / \rho A$ and $w(x, t)=\phi(x) T(t)$.

The shape function $\phi(x)$ is given by

$$
\begin{aligned}
\phi(x)= & C_{1} \sin (\beta x)+C_{2} \cos (\beta x) \\
& +C_{3} \sinh (\beta x)+C_{4} \cosh (\beta x),
\end{aligned}
$$

where $C_{p}, p=1, \ldots, 4$, are constants and $\beta$ is related to the natural frequency through

$$
\beta_{n}^{4}=\frac{\omega_{n}^{2}}{c^{2}} \Longrightarrow \omega_{n}=\beta_{n}^{2} \sqrt{\frac{E I}{\rho A}} .
$$

If we consider a set of boundary conditions, Eqn. (14) leads to an eigenproblem, whose eigenvalues are $\beta_{n} l, n=1,2, \ldots, N$, and the elements of the respective eigenvectors are the constants $C_{p}^{(n)}$. From the eigenvalues, the natural frequencies are calculated and, from the eigenvectors, the mode shapes are defined as,

$$
\begin{aligned}
\phi_{n}(x)= & C_{1}^{(n)} \sin \left(\beta_{n} x\right)+C_{2}^{(n)} \cos \left(\beta_{n} x\right) \\
& +C_{3}^{(n)} \sinh \left(\beta_{n} x\right)+C_{4}^{(n)} \cosh \left(\beta_{n} x\right) .
\end{aligned}
$$

Note that each eigenvector has infinite solutions, being functions of one of the constants $C_{p}^{(n)}$. Hence, we must give one extra equation in order to establish a single solution for the eigenvectors, e.g., satisfy a prescribed displacement at a given position of the beam.

If a structure presents discontinuities between its boundaries, such as discs, supports or others, we must define the shape function of Eqn. (16) as a piecewise function with $h+1$ sections, $h$ being the number of discontinuities. Hence, Eqn. (16) is recast as

$$
\begin{aligned}
& \left(\phi_{n}(x)\right)_{\alpha} \\
& =C_{4 \alpha-3}^{(n)} \sin \left(\beta_{n} x\right)+C_{4 \alpha-2}^{(n)} \cos \left(\beta_{n} x\right) \\
& \quad+C_{4 \alpha-1}^{(n)} \sinh \left(\beta_{n} x\right)+C_{4 \alpha}^{(n)}, \cosh \left(\beta_{n} x\right)
\end{aligned}
$$

for $x \in\left[x_{h_{\alpha-1}}, x_{h_{\alpha}}\right]$, with $\alpha=1, \ldots, h+1$. Note that, for $\alpha=1$ we have $x_{h_{0}}=0$ and for $\alpha=h+1$ we get $x_{h_{h+1}}=l$, i.e., the left and right end sides of the beam, respectively.

As mentioned above, the shape functions must satisfy the set of boundary conditions defined for a certain problem. Moreover, if the shape function is piecewise one, we must impose a set of continuity conditions as well. Both boundary and continuity conditions are described in detail below. Figure 1 gives an illustrative example of a generic beam model with $h$ discontinuities.

2.3. Boundary conditions. Two types of generic sets of boundary conditions are presented. 
2.3.1. Generic elastic support. If we do not consider any assumptions on the stiffness parameters of the support, we have $k_{i} \geq 0$ and $k_{t_{j}}>0$, which leads to the following set of boundary conditions at $x=0$ :

$$
\begin{aligned}
\left.\frac{\partial^{2}}{\partial x^{2}}(E(x) I(x) w(x, t))\right|_{x=0} & =\left.k_{t_{j}} \frac{\partial}{\partial x} w(x, t)\right|_{x=0}, \\
\left.\frac{\partial^{3}}{\partial x^{3}}(E(x) I(x) w(x, t))\right|_{x=0} & =-\left.k_{i} w(x, t)\right|_{x=0}
\end{aligned}
$$

and, at $x=l$,

$$
\begin{aligned}
& \left.\frac{\partial^{2}}{\partial x^{2}}(E(x) I(x) w(x, t))\right|_{x=l}=-\left.k_{t_{j}} \frac{\partial}{\partial x} w(x, t)\right|_{x=l} \\
& \left.\frac{\partial^{3}}{\partial x^{3}}(E(x) I(x) w(x, t))\right|_{x=l}=\left.k_{i} w(x, t)\right|_{x=l}
\end{aligned}
$$

From the set of boundary conditions for a generic elastic support it is possible to derive all the sets of boundary conditions for supported ends. A specific application will be given in the experimental application section.

2.3.2. Generic disc. If we consider a disc attached at the tip of a beam, we have $J_{s} \geq 0$ and $m_{r}>0$, which leads to the following set of boundary conditions at $x=0$

$$
\begin{aligned}
\left.\frac{\partial^{2}}{\partial x^{2}}(E(x) I(x) w(x, t))\right|_{x=0} & =\left.J_{s} \frac{\partial^{3}}{\partial x \partial t^{2}} w(x, t)\right|_{\substack{x=0 \\
(22)}}, \\
\left.\frac{\partial^{3}}{\partial x^{3}}(E(x) I(x) w(x, t))\right|_{x=0} & =-\left.m_{r} \frac{\partial^{2}}{\partial t^{2}} w(x, t)\right|_{x=0}
\end{aligned}
$$

and, at $x=l$,

$$
\begin{aligned}
\left.\frac{\partial^{2}}{\partial x^{2}}(E(x) I(x) w(x, t))\right|_{x=l} & =-\left.J_{s} \frac{\partial^{3}}{\partial x \partial t^{2}} w(x, t)\right|_{\substack{x=l \\
(24)}} \\
\left.\frac{\partial^{3}}{\partial x^{3}}(E(x) I(x) w(x, t))\right|_{x=l} & =\left.m_{r} \frac{\partial^{2}}{\partial t^{2}} w(x, t)\right|_{x=l} .
\end{aligned}
$$

From the set of boundary conditions for a generic disc it is possible to derive all the sets of boundary conditions for discs at both the ends of the beam. A specific application will be given in the experimental application section.

2.4. Continuity conditions. In the case of intermediate material or geometrical discontinuities, the shape function must be defined as a piecewise one and we must impose a set of continuity conditions or equations for each discontinuity between beam sections $h$ and $h+1$ at $\left.x_{h_{\alpha-1}} \in\right] 0, l[$, with $\alpha=2, \ldots, h+1$. Hence, we have to satisfy

$$
\begin{gathered}
\left.(E(x) I(x) w(x, t))_{h}\right|_{x=x_{h_{\alpha-1}}} \\
=\left.(E(x) I(x) w(x, t))_{h+1}\right|_{x=x_{h_{\alpha-1}}}, \\
\left.\frac{\partial}{\partial x}(E(x) I(x) w(x, t))_{h}\right|_{x=x_{h_{\alpha-1}}} \\
=\left.\frac{\partial}{\partial x}\left((E(x) I(x) w(x, t))_{h+1}\right)\right|_{x=x_{h_{\alpha-1}}}
\end{gathered}
$$

and one of the following sets of equations, depending on the discontinuity type.

\subsubsection{Generic elastic support.}

$$
\begin{gathered}
\left.\frac{\partial^{2}}{\partial x^{2}}(E(x) I(x) w(x, t))_{h}\right|_{x=x_{h_{\alpha-1}}} \\
\quad+\left.k_{t_{j}} \frac{\partial}{\partial x}(w(x, t))_{h}\right|_{x=x_{h_{\alpha-1}}} \\
=\left.\frac{\partial^{2}}{\partial x^{2}}(E(x) I(x) w(x, t))_{h+1}\right|_{x=x_{h_{\alpha-1}}}, \\
\left.\frac{\partial^{3}}{\partial x^{3}}(E(x) I(x) w(x, t))_{h}\right|_{x=x_{h_{\alpha-1}}} \\
\quad-\left.k_{i}(w(x, t))_{h}\right|_{x=x_{h_{\alpha-1}}} \\
=\left.\frac{\partial^{3}}{\partial x^{3}}(E(x) I(x) w(x, t))_{h+1}\right|_{x=x_{h_{\alpha-1}}} .
\end{gathered}
$$

As mentioned in the case of boundary conditions, from this set of equations it is possible to derive all the continuity equations regarding an intermediate support, from clamped to simply supported, at location $x=x_{h_{\alpha-1}}$.

\subsubsection{Generic disc.}

$$
\begin{gathered}
\left.\frac{\partial^{2}}{\partial x^{2}}(E(x) I(x) w(x, t))_{h}\right|_{x=x_{h_{\alpha-1}}} \\
\quad+\left.J_{s} \frac{\partial^{3}}{\partial x \partial t^{2}}(w(x, t))_{h}\right|_{x=x_{h_{\alpha-1}}} \\
=\left.\frac{\partial^{2}}{\partial x^{2}}(E(x) I(x) w(x, t))_{h+1}\right|_{x=x_{h_{\alpha-1}}}, \\
\left.\frac{\partial^{3}}{\partial x^{3}}(E(x) I(x) w(x, t))_{h}\right|_{x=x_{h_{\alpha-1}}} \\
-\left.m_{r} \frac{\partial^{2}}{\partial t^{2}}(w(x, t))_{h}\right|_{x=x_{h_{\alpha-1}}} \\
=\left.\frac{\partial^{3}}{\partial x^{3}}(E(x) I(x) w(x, t))_{h+1}\right|_{x=x_{h_{\alpha-1}}} .
\end{gathered}
$$

From this set of equations it is also possible to derive all the continuity equations regarding a material or a geometric intermediate discontinuity at location $x=$ $x_{h_{\alpha-1}}$, e.g., a disc or a rotor with a given mass and inertia or even an abrupt change in the cross section of the beam. 


\section{Experimental application}

To consolidate the above presented formulation for the bending vibration problem of a Bernoulli-Euler beams, we use the structure of Fig. 2 as an experimental case study. As can be seen, it is a didactic rotating machine with a elastically supported shaft carrying a disc between supports. The material and geometric properties of the shaft are $E=73 \mathrm{GPa}, I=1.277 \times 10^{-9} \mathrm{~m}^{4}, \rho=$ $2766 \mathrm{~kg} \cdot \mathrm{m}^{-3}$ and $A=1.2668 \times 10^{-4} \mathrm{~m}^{2}$. Note that the beam is uniform and therefore the following properties are constants: $E(x)=E, I(x)=I, \rho(x)=\rho$ and $A(x)=A$.

The equipment studied has an attached disc between supports. Hence, it has one intermediate discontinuity, which leads to $h=1$. The referred disc mass and rotational inertia are $m=3 \mathrm{~kg}$ and $J=2.8 \times$ $10^{-3} \mathrm{~kg} \cdot \mathrm{m}^{2}$, respectively.

Summarizing, in the present case study, we consider that the structure of Fig. 2 can be accurately modelled as a beam on elastic supports with $k_{t_{j}}>0$ and $k_{i}=\infty$, carrying a disc at $x=x_{h_{1}}$, with $x_{h_{1}}$ as the coordinate of the only intermediate discontinuity. Thus, we must consider $\phi_{n}(x)$ as a piecewise function with two branches (Eqn. (17) with $\alpha=1$ ) and impose the following set of boundary and continuity conditions of Eqns. (32)- 38):

$$
\begin{gathered}
\left(\phi_{n}(0)\right)_{1}=\left(\phi_{n}(l)\right)_{2}=0, \\
\left.E I\left(\phi_{n}^{\prime \prime}(x)\right)_{1}\right|_{x=0}=\left.k_{t_{1}}\left(\phi_{n}^{\prime}(x)\right)_{1}\right|_{x=0}, \\
\left.E I\left(\phi_{n}^{\prime \prime}(x)\right)_{2}\right|_{x=l}=-\left.k_{t_{2}}\left(\phi_{n}^{\prime}(x)\right)_{2}\right|_{x=l}, \\
\left(\phi\left(x_{h_{1}}\right)\right)_{1}=\left(\phi\left(x_{h_{1}}\right)\right)_{2}, \\
\left.\left(\phi^{\prime}(x)\right)_{1}\right|_{x=x_{h_{1}}}=\left.\left(\phi^{\prime}(x)\right)_{2}\right|_{x=x_{h_{1}}}, \\
\left.E I\left(\phi_{n}^{\prime \prime}(x)\right)_{1}\right|_{x=x_{h_{1}}}-\left.\omega_{n}^{2} J\left(\phi_{n}^{\prime}(x)\right)_{1}\right|_{x=x_{h_{1}}} \\
=\left.E I\left(\phi_{n}^{\prime \prime}(x)\right)_{2}\right|_{x=x_{h_{1}}}, \\
=\left.E I\left(\phi_{n}^{\prime \prime \prime}(x)\right)_{2}\right|_{x=x_{h_{1}}} .
\end{gathered}
$$

Finally, Rayleigh's quotient for this kind of structure has the following form:

$$
\begin{aligned}
R_{n}= & \omega_{n}^{2} \\
= & {\left[E I \int_{0}^{l}\left(\phi_{n}^{\prime \prime}(x)\right)^{2} \mathrm{~d} x+\left.\sum_{j} k_{t_{j}} \phi_{n}^{\prime}(x)\right|_{x=x_{j}} ^{2}\right] } \\
& \times\left[\rho A \int_{0}^{l} \phi_{n}^{2}(x) \mathrm{d} x+\left.m \phi_{n}(x)\right|_{x=x_{h_{1}}} ^{2}\right. \\
& \left.+\left.J \phi_{n}^{\prime}(x)\right|_{x=x_{h_{1}}} ^{2}\right]^{-1},
\end{aligned}
$$

with $x \in[0, l]$ and $x_{j}=\left[\begin{array}{ll}0 & l\end{array}\right]^{T}$.

Several impact tests were performed on the presented test rig and the obtained experimental fundamental frequency $\left(\left.f_{1}\right|_{X}=38.907 \mathrm{~Hz}\right.$ ) is used to set the objective function for the optimization problem, which is a function of $\phi_{1}(x)$, the approximated first mode shape.

Regarding the available experimental data, concerning the set up of Fig. 2, results for the case where the disc is placed at the mid-span are presented in this paper. However, from the theoretical background (Section 2), other configurations can be considered.

\section{Optimal identification of the support parameters}

Considering the complexity of real physical systems, traditional gradient based optimizations techniques can easily lead to non-global optimal solutions. This may be due to non-linear/non-convex design spaces and thus the use of global optimization approaches, namely, nature-inspired optimization techniques, should be addressed.

Within the framework of nature-inspired optimization methods, one can find techniques such as genetic algorithms, particle swarm optimization, ant colonies, or differential evolution, among others (Eiben and Smith, 2003). Taking into account the DE performance when compared with other evolutionary strategies (Das and Suganthan, 2011) and the experience of the authors in this field (Loja et al., 2010; 2013; 2014; Silva and Loja, 2013; Loja, 2014), in the present work we have chosen the use of DE.

4.1. Differential evolution fundamentals. The DE algorithm was developed by Storn and Price (1997). DE is a population-based optimization technique (Babu and Jehan, 2003) that uses difference vectors in order to generate trial individuals for the next generation. The referred difference vectors are computed from randomly selected members of the population. For each population, the objective function is evaluated for each member, in order to find the best population member until a termination criterion is achieved. 
DE congregates characteristics from well-known global optimization and meta-heuristics algorithms. Although it is a global search technique, DE preserves a search direction vector, which gives an important descent property, and the randomness of populations, which improves the method's robustness (Kitayama et al., 2011). The available algorithmic schemes differ according to the number of computed difference vectors and whether the current individual or the global best one will be used or not as part of the mutation. Hence, it is usual to designate a DE scheme by the following codes: DE/Vector/n.Vectors/Cross.Sch., where Vector specifies the population member to be mutated, $n$.Vectors specifies the number of computed difference vectors and Cross.Sch. specifies the used crossover scheme.

Babu and Munawar (2007) indicated DE/best/1/bin as one of the most promising DE strategies, although the best scheme remains problem dependent and the authors find $\mathrm{DE} / \mathrm{best} / 2$ more effective for the type of problems treated in this work. Recent developments regarding DE optimization techniques and applications can be found in the works of Price et al. (2005), Chakraborty (2008) and Martinović et al. (2014). Only few applications of DE to structural engineering can be found, for instance, the ones of Savoia and Vincenzi (2008) or Reed et al. (2013). In the work of Savoia and Vincenzi (2008), DE is used to identify mass and stiffness properties of civil structures from dynamical response data. The robustness of the algorithm to the variability of test responses is also evaluated, whereas in the work of Reed et al. (2013) a modified version of the DE algorithm, specifically tailored to structural identification problems, is proposed. The authors also compared the recommended algorithm with the one of Storn and Price (1997) and showed a faster convergence for the problem of damage identification of a submerged structure.

In the present work, despite several schemes tried, we use the DE/best/1 and DE/best/2 optimization strategies, where best specifies the vector of lowest objective function value from the current population to be mutated and 1 and 2 are the number of difference vectors used for calculation. Note that an adaptive mutation (AM) is considered and, as the floating point representation is used, the crossover step is performed by single arithmetic recombination of each population member (Eiben and Smith, 2003).

4.2. Optimal design problem. Considering the structure described in the experimental application section, we can state an optimization problem as

$$
\min \Omega_{n}=\min \left\|\frac{\left.\omega_{n}^{2}\right|_{X}-R_{n}\left(\phi_{n}\left(x, k_{t_{j}}\right)\right)}{\left.\omega_{n}^{2}\right|_{X}}\right\|_{2}
$$

subject to

$$
k_{t_{j}}^{\text {low }} \leq k_{t_{j}} \leq k_{t_{j}}^{u p}, \quad j=1,2,
$$

where $\Omega_{n}$ is the objective function related to the $n$ eigenfrequency, $k_{t_{j}}$ is the vector of design variables, whose values are bounded by the lower and upper limits $k_{t_{j}}^{\text {low }}$ and $k_{t_{j}}^{u p}$, respectively. In this context, the design variables to be identified are the torsional stiffness coefficients and the imposed limits are used as boundary constraints for the optimization process. The objective function is the euclidean norm of the relative difference between the experimental fundamental frequency and its analytical approximation, given by Eqn. (40).

4.3. Kriging predictor as a meta-model. Usually, global optimization techniques, such as DE, are computationally expensive. They require hundreds or even thousands of evaluations of the objective function, which, in the present case, encompass the evaluation of an eigenproblem. Meta-models, or the so-called fast-running surrogates, have been successfully used to describe the relationship between the model responses (here the fundamental eigenfrequency) and design parameters with a significant decrease in the computational effort. Amongst available meta-models, the Kriging ones are excellent predictors of model responses (De Munck et al., 2009). The Kriging predictor is a Gaussian process model (Rasmussen and Williams, 2005), and therefore it produces unbiased estimates at the reference or training locations, i.e., despite the quality of the model responses approximation, one can be certain that the model responses at reference locations are not degraded.

In the current work, we build a Kriging model based on a set of design parameters, torsional stiffness coefficients, and their responses, computed using Rayleigh's quotient. We consider the covariance function of the Ornstein-Uhlenbeck process (Rasmussen and Williams, 2005). Hence, the Kriging response prediction $\hat{z}$ may be written as

$$
\hat{z}=\lambda^{T} \mathbf{z}
$$

where $\mathbf{z}$ is the vector of reference responses used for training the Kriging model and $\boldsymbol{\lambda}$ is the vector of regression coefficients, computed from the selected covariance function as

$$
\boldsymbol{\lambda}=\left[\begin{array}{cccc}
\operatorname{cov}_{\mathbf{u}_{1}, \mathbf{u}_{1}} & \cdots & \operatorname{cov}_{\mathbf{u}_{1}, \mathbf{u}_{n_{s}}} & 1 \\
\vdots & \ddots & \vdots & \vdots \\
\operatorname{cov}_{\mathbf{u}_{n_{s}}, \mathbf{u}_{1}} & \cdots & \operatorname{cov}_{\mathbf{u}_{n_{s}}, \mathbf{u}_{n_{s}}} & 1 \\
1 & \cdots & 1 & 0
\end{array}\right]^{-1}\left[\begin{array}{c}
\operatorname{cov}_{\mathbf{u}_{1}, \hat{\mathbf{u}}} \\
\vdots \\
\operatorname{cov}_{\mathbf{u}_{n_{s}}, \hat{\mathbf{u}}} \\
1
\end{array}\right]
$$

where cov., is the covariance function of the Ornstein-Uhlenbeck process, $\mathbf{u}$ is the vector of each one of the $n_{s}$ reference design parameters or training 
locations, and $\hat{\mathbf{u}}$ is the vector of design parameters for which the Kriging prediction is performed.

Summarizing, we build the Kriging model and use its prediction $\hat{z}$ on each evaluation of the objective function by the DE algorithm. Hence, Eqn. (40) is recast as

$$
\min \Omega_{n}=\min \left\|\frac{\left.\omega_{n}^{2}\right|_{X}-\hat{z}\left(k_{t_{j}}\right)}{\left.\omega_{n}^{2}\right|_{X}}\right\|_{2} .
$$

Note that the optimization process is bounded by the lower and upper limits of the design parameters (Eqn. (41)). In the present paper, we use fixed side constraints for $k_{t_{j}}$ : $k_{t_{j}} \in[500,5000] \mathrm{N} / \mathrm{m}$. These bounds were defined in order to improve convergence, after wider ranges had been tried.

4.4. Optimal identification procedure. The proposed procedure to identify the torsional stiffness coefficients on structures modelled as an elastically restrained Bernoulli-Euler beam is implemented in the symbolic computation commercial software Maple (Maplesoft, 2010) following the steps of Algorithm 1

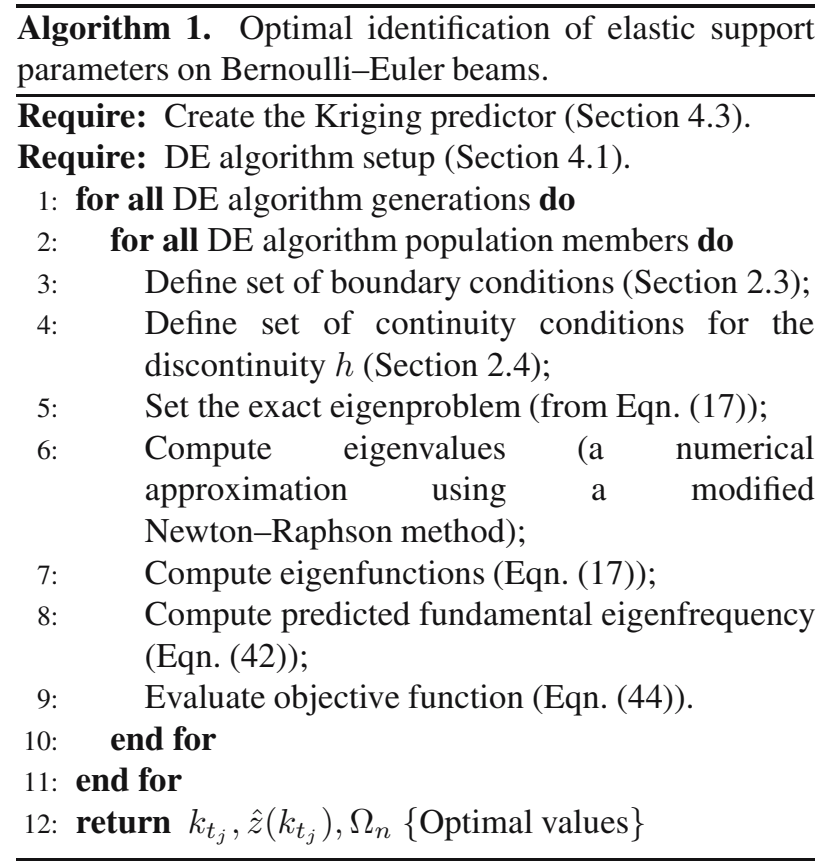

Note that the adopted stopping criterion is only the defined DE maximum number of generations. No tolerance on the solution convergence is considered due to the fact that we wanted to overview the overall evolution of the optimization process. The results presented in Section 5.3 support this decision. As expected, due to the employed DE scheme, a good convergence performance is achieved (Fig. 8).

For solution verification purposes, after the application of the described optimal identification procedure, we use the Rayleigh quotient of Eqn. 39) to compute the exact solution of the problem in order to assess the quality of the identified stiffness coefficients, as it is still a function of the Kriging predictor quality.

\section{Results and discussion}

5.1. Solutions for the mode shapes. In the work of Silva and Maia (2013), the bending vibration problem of Bernoulli-Euler beams was presented and the influence of the disc position in the mode shape highlighted.

In the present work, the exact solution for the model of Fig. 3 is implemented recurring to the symbolic computation commercial software Maple (Maplesoft, 2010). The symbolic implementation of the presented problem has several computational advantages over the previous numerical one, both in terms of programming and the range of solutions, i.e., it is now possible to change every single design parameter without the need to explicitly evaluate every single shape function and respective derivatives beforehand.

Figures 46 show a set of solutions for different models obtained from a unique computer code. The influence of the type of support (represented by a black diamond) and the disc location (represented by a black circle) on the mode shape is clearly understandable from these figures.

5.2. Kriging predictions. As mentioned, a Kriging model was created in order to be used as a meta-model, avoiding the evaluation of the exact problem solutions for each member of each DE generation, at a satisfactory accuracy. Figure 7 shows the Kriging model surface of predictions and its mean squared error. Note that the Kriging model was trained with a data point selected from preliminary runs of different DE configurations, using the exact solution evaluation, and some other data

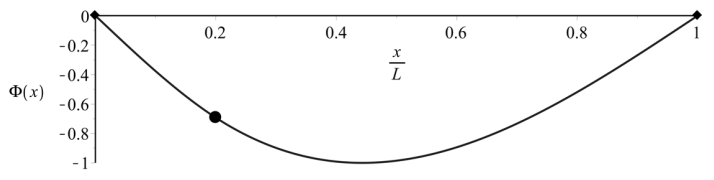

(a)

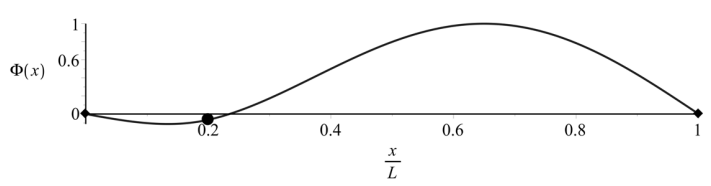

(b)

Fig. 4. Mode shapes for a simply supported beam with a disc at $x / l=0.2$ (supports: black diamond, disc: black circle): first mode shape (a), second mode shape (b). 


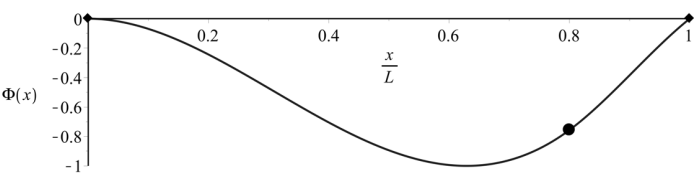

(a)

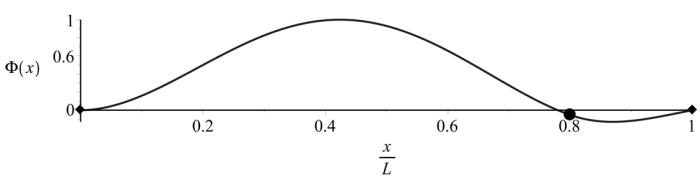

(b)

Fig. 5. Mode shapes for a beam with the left end side clamped, the right end side on an elastic support $\left(k_{t_{2}}=500 \mathrm{~N} / \mathrm{m}\right.$ and $k_{2}=\infty$ ) and a disc at $x / l=0.8$ (supports: black diamond, disc: black circle): first mode shape (a), third mode shape (b).

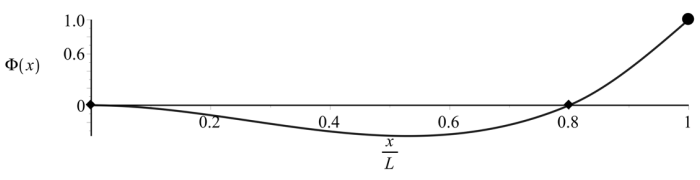

(a)

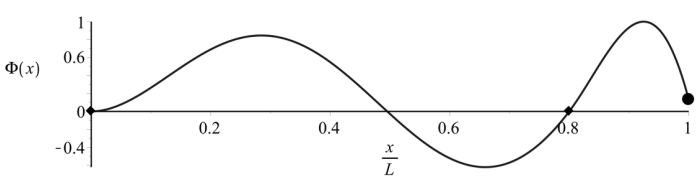

(b)

Fig. 6. Mode shapes for a beam with the left end side clamped, an intermediate elastic support at $x / l=0.8\left(k_{t_{2}}=\right.$ $500 \mathrm{~N} / \mathrm{m}$ and $k_{2}=\infty$ ) and a disc at the right end side (supports: black diamond, disc: black circle): first mode shape (a), third mode shape (b).

points randomly selected within the feasible parameters space. This training data points are represented in Fig. 7 as black dots. In Fig. 7(a) the plane corresponding to the experimental fundamental frequency is shown (in grey) and some points (black dots) on the contour line of the experimental fundamental frequency are depicted in Fig. 7(b). Note that this isoline marks the interception of the experimental fundamental frequency plan with the surface of predictions.

The Kriging predictor, whose results are presented in Fig. 7 is the one used to perform the optimal identification of the torsional stiffness coefficients of the elastic supports. Note that unbiased estimates at the reference or training locations are guaranteed and thus it is evident that the error at such locations is null (Fig. 7(c)).
Table 1. Comparison of computational times: one run of the exact solution (Rayleigh's quotient) (a), one run of DE for one generation with two members using the Kriging predictor (b), one run of DE for one generation with 100 members using the Kriging predictor (c).

\begin{tabular}{|c|c|c|}
\hline Run & CPU time (s) & CPU time ratio \\
\hline \hline (a) & 45 & 1.00 \\
\hline (b) & 1.826 & 0.04 \\
\hline (c) & 19.905 & 0.44 \\
\hline
\end{tabular}

Recalling the purpose of the Kriging predictor, which aims at decreasing the computational effort without a significant loss in the accuracy of the identification process, Table 1 shows a comparison of computational times, in seconds, with the computational time ratios calculated with respect to the time spent to complete a single evaluation of the exact solution (Rayleigh's quotient).

Note that the same DE configuration was used to compute the results (b) and (c) of Table 1 (static mutation for one difference vector). It is worth emphasizing that computing the result of (b) is equivalent to computing the exact solution twice. Therefore, if Rayleigh's quotient had been used to compute the objective function for each member of a usual DE population size, instead of the Kriging predictor, the computational time would have easily reached several hours, in comparison with a few minutes spent by the described DE implementation.

5.3. Optimal identification of elastic supports parameters. In order to identify the most suitable configuration for the DE algorithm, a parametric study on the DE algorithm was conducted. Hence, several sets of DE parameters were tried. Note that each set of DE parameters is an arrangement of different variables, namely, the number of difference vectors used in the mutation step Dif, the perturbation factor $F$ (if adaptive mutation with generations, define lower and upper bounds, $F_{i}$ and $F_{f}$ ), the population size $N_{p}$, and the maximum number of generations to be evaluated g. Moreover, the adopted DE strategy does not need a crossover constant, as we use a single arithmetic recombination process with a random parameter for each one of the population members.

In the present paper, a selection of the best results from the parametric study is presented (Table 2). Note that $\Omega_{n}$ corresponds to the optimum value of the objective function at the last generation, computed from Eqn. (44) with $\left.f_{1}\right|_{X}$ for the DE optimal members, $k_{t_{1}}$ and $k_{t_{2}}$. As referred, the following side constraint parameters were adopted: $k_{t_{j}}^{\text {low }}=500 \mathrm{~N} / \mathrm{m}$ and $k_{t_{j}}^{u p}=5000 \mathrm{~N} / \mathrm{m}$. Also in Table 2, the first column identifies the DE strategy considered, which is the same as in Table 3, and AM 


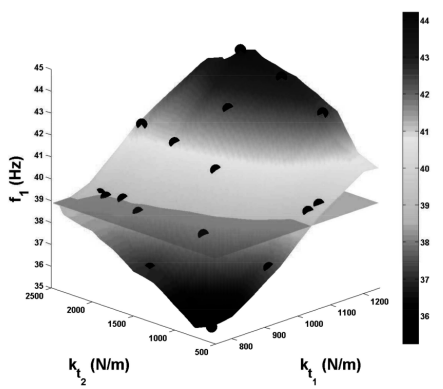

(a)

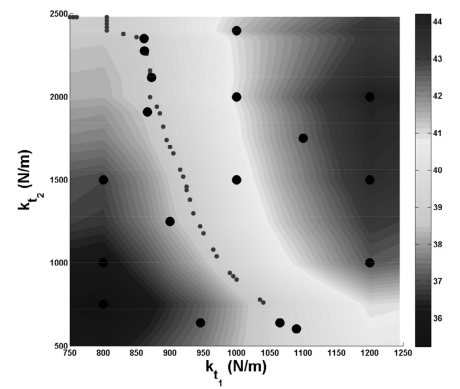

(b)

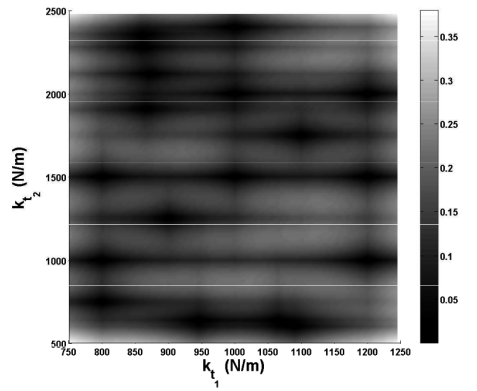

(c)

Fig. 7. Kriging predictor results: surface of prediction with the experimental fundamental frequency plane and the training data (black circles) (a), $k_{t_{1}}$ vs. $k_{t_{2}}$ planar or view of the surface of prediction for the training data (black circles) and the experimental fundamental frequency isoline (black dots) (b), and the mean squared error of the Kriging predictor (c).

Table 2. DE parametric study and respective results $\left(\left.f_{1}\right|_{X}=38.907 \mathrm{~Hz}\right)$.

\begin{tabular}{|c|c|c|c|c|c|c||c|c|c|c|}
\hline Strategy & AM & Dif. & $F_{i}$ & $F_{f}$ & $N_{p}$ & $g$ & $k_{t_{1}}(\mathrm{~N} / \mathrm{m})$ & $k_{t_{2}}(\mathrm{~N} / \mathrm{m})$ & $\begin{array}{c}f_{1}(\mathrm{~Hz}) \\
\text { (Eqn. (42) })\end{array}$ & $\begin{array}{c}\Omega_{n} \\
\text { (Eqn. (44) })\end{array}$ \\
\hline \hline (a) & 1 & 2 & 1 & 0.01 & 10 & 50 & 807.6215 & 3974.4138 & 38.906998 & $5.9302 \mathrm{E}-08$ \\
\hline (b) & 1 & 2 & 1 & 0.01 & 20 & 50 & 749.5921 & 3831.7455 & 38.907000 & $5.2445 \mathrm{E}-09$ \\
\hline (c) & 1 & 2 & 1 & 0.01 & 20 & 200 & 763.9674 & 3845.8196 & 38.907000 & $1.8230 \mathrm{E}-09$ \\
\hline (d) & 0 & 2 & 2 & n.a. & 20 & 50 & 858.0000 & 4294.0000 & 38.910427 & $8.8091 \mathrm{E}-05$ \\
\hline
\end{tabular}

stands for adaptive mutation ( 1 if it is used or 0 otherwise).

Table 3. Exact solution results for the optimal torsional stiffness coefficients and the error with respect to the experimental fundamental frequency $\left(\left.f_{1}\right|_{X}=38.907 \mathrm{~Hz}\right)$.

\begin{tabular}{|c|c|c|c|c|}
\hline Strategy & $\begin{array}{c}k_{t_{1}} \\
(\mathrm{~N} / \mathrm{m})\end{array}$ & $\begin{array}{c}k_{t_{2}} \\
(\mathrm{~N} / \mathrm{m})\end{array}$ & $\begin{array}{c}R_{1}(\mathrm{~Hz}) \\
(\text { Eqn. (39) })\end{array}$ & error $(\%)$ \\
\hline \hline (a) & 807.62 & 3974.41 & 38.731 & 0.45 \\
\hline (b) & 749.59 & 3831.75 & 37.620 & 3.31 \\
\hline (c) & 763.97 & 3845.82 & 37.891 & 2.61 \\
\hline (d) & 858.00 & 4294.00 & 39.728 & -2.11 \\
\hline
\end{tabular}

After estimating the optimal torsional stiffness coefficients, we must compute the exact solution (Eqn. (39)), in order to assess the accuracy of the optimal design parameters computed with the Kriging predictor. In Table 3. one can find results for the exact solution and its error with respect to the experimental fundamental frequency $\left(\left.f_{1}\right|_{X}=38.907 \mathrm{~Hz}\right)$. The accuracy of the obtained results is excellent. However, the results for all the presented DE strategies suggest an asymmetric structure, which is not expected at a first glance, although the experimental test rig is in fact asymmetric. Note that in the experimental structure (Fig. 2) the shaft under study is not isolated from the one of the electrical motor, as considered on the theoretical model of Fig. 3 i.e., the real shaft is not confined between its supports.

The evolution for the best computed solution (strategy (a): Table 3) and its corresponding first mode shape of the exact problem with the identified optimal torsional stiffness coefficients are presented in Figs. 8 and 9, respectively. Note that the so-called strategy (a) does not attain the lowest cost for the objective function computed with the Kriging predictor (Table 2), although the identified optimal torsional stiffness coefficients of strategy (a) led to the minimum error, regarding the relative difference between the fundamental experimental frequency and the one approximated by Rayleigh's quotient (Table 3).

It is relevant to note that this hybrid procedure yields good convergence characteristics, requiring a low number of generations to achieve good results, as we can globally observe in Fig. 8(a) Additionally, Fig. 8(b) provides complementary information that allows concluding on the adaptive mutation scheme influence. The adopted mutation scheme enables fine tuning of the obtained global optimum, considering the pre-defined maximum number of generations.

\section{Conclusion}

The bending vibration problem of Bernoulli-Euler beams is addressed. A comprehensive formulation for the referred subject and its computational implementation are presented. Some examples of hypothetical structures are given. Besides the classical beam theory, the authors introduce a hybrid identification procedure in 


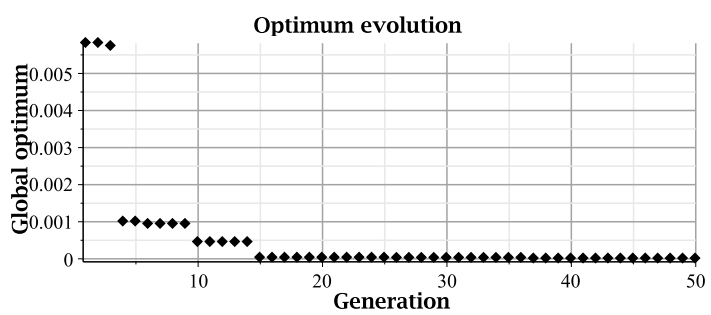

(a)

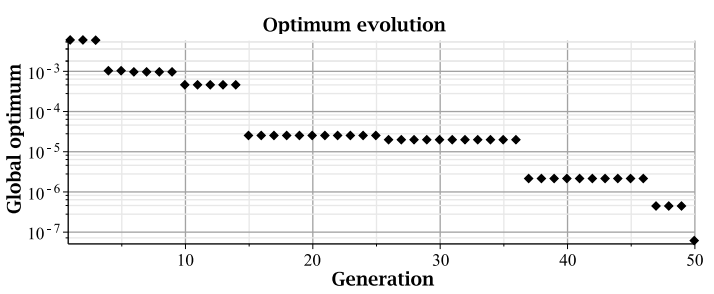

(b)

Fig. 8. DE global optimum evolution (strategy (a): Table 3): linear scale (a), logarithmic scale (b).

order to identify the torsional stiffness coefficients of the supports that experimentally validate the analytical solution. The computed optimal torsional stiffness coefficients lead to a very accurate solution, in terms of the predicted eigenfrequency, with a relative error under $0.5 \%$, which supports the use of the Kriging predictor as a meta-model. In fact, if one considers the proposed hybrid identification procedure without using a meta-model, it is computationally prohibitive. The optimal solutions indicate that the structure is not symmetric, which is true in reality but is not considered in the analytical model. Therefore, a new experimental test with a decoupled shaft and a correspondent analytical model must be addressed in future work.

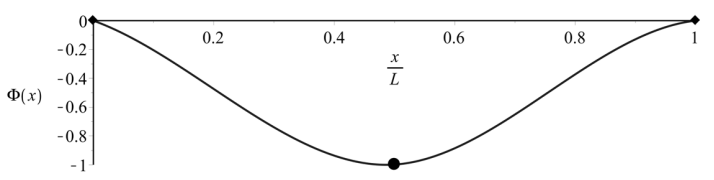

Fig. 9. First mode shape for the model with optimal torsional stiffness coefficients (strategy (a): Table 3) (supports: black diamond, disc: black circle)

\section{Acknowledgment}

The authors acknowledge the support of the Portuguese Foundation for Science and Technology, FCT, under the projects PEst/OE/EME/LA0022/2011 and PTDC/ATP-AQI/5355/2012, and the Ph.D. grant SFRH/BD/44696/2008.

\section{References}

Ahmadian, H., Mottershead, J.E. and Friswell, M.I. (2001). Boundary conditions identification by solving characteristic equations, Journal of Sound and Vibration 247(5): 755-763.

Albarracín, C., Zannier, L. and Grossi, R. (2004). Some observations in the dynamics of beams with intermediate supports, Journal of Sound and Vibration 271(1-2): 475-480.

Auciello, N. (1996). Transverse vibrations of a linearly tapered cantilever beam with tip mass of rotary inertia and eccentricity, Journal of Sound and Vibration 194(1): 25-34.

Babu, B. and Jehan, M. (2003). Differential evolution for multi-objective optimization, Proceedings of the 2003 Congress on Evolutionary Computation, CEC 2003, Canberra, Australia, pp. 2696-2703.

Babu, B. and Munawar, S. (2007). Differential evolution strategies for optimal design of shell-and-tube heat exchangers, Chemical Engineering Science 62(14): 3720-3739.

Bashash, S., Salehi-Khojin, A. and Jalili, N. (2008). Forced vibration analysis of flexible Euler-Bernoulli beams with geometrical discontinuities, Proceedings of the American Control Conference, ACC 2008, Seattle WA, USA, pp. 4029-4034.

Biondi, B. and Caddemi, S. (2005). Closed form solutions of Euler-Bernoulli beams with singularities, International Journal of Solids and Structures 42(9-10): 3027-3044.

Biondi, B. and Caddemi, S. (2007). Euler-Bernoulli beams with multiple singularities in the flexural stiffness, European Journal of Mechanics, A: Solids 26(7): 789-809.

Chakraborty, U.K. (2008). Advances in Differential Evolution, 1st Edition, Springer, New York, NY.

Chesne, S. (2012). Identification of beam boundary conditions using displacement derivatives estimations, Proceedings of the 16th IFAC Symposium on System Identification, Brussels, Belgium, pp. 416-421.

Das, S. and Suganthan, P.N. (2011). Differential evolution: A survey of the state-of-the-art, IEEE Transactions on Evolutionary Computation 15(1): 4-31.

De Munck, M., Moens, D., Desmet, W. and Vandepitte, D. (2009). An efficient response surface based optimisation method for non-deterministic harmonic and transient dynamic analysis, Computer Modeling in Engineering \& Sciences 47(2): 119-166.

De Rosa, M. and Auciello, N. (1996). Free vibrations of tapered beams with flexible ends, Computers \& Structures 60(2): 197-202.

De Rosa, M., Franciosi, C. and Maurizi, M. (1996). On the dynamic behaviour of slender beams with elastic ends carrying a concentrated mass, Computers \& Structures 58(6): 1145-1159.

Eiben, A.E. and Smith, J.E. (2013). Introduction to Evolutionary Computing, Natural Computing Series, Springer-Verlag, Heidelberg. 
Elishakoff, I. and Pentaras, D. (2006). Apparently the first closed-form solution of inhomogeneous elastically restrained vibrating beams, Journal of Sound and Vibration 298(1-2): 439-445.

Grossi, R. and Albarracín, C. (2003). Eigenfrequencies of generally restrained beams, Journal of Applied Mathematics 2003(10): 503-516.

Kitayama, S., Arakawa, M. and Yamazaki, K. (2011). Differential evolution as the global optimization technique and its application to structural optimization, Applied Soft Computing 11(4): 3792-3803.

Lin, S.C. and Hsiao, K.M. (2001). Vibration analysis of a rotating Timoshenko beam, Journal of Sound and Vibration 240(2): 303-322.

Loja, M.A.R., Barbosa, J.I. and Mota Soares, C.M. (2010). Optimal design of piezolaminated structures using b-spline strip finite element models models and genetic algorithms, International Journal for Computational Engineering Science and Mechanics 11(4): 185-195.

Loja, M.A.R., Silva, T.A.N., Barbosa, I.J.C. and Simes, C.N.F. (2013). Analysis and optimization of agro-waste composite beam structures, Usak University Journal of Material Sciences 2(1): 45-60.

Loja, M.A.R., Mota Soares, C.M. and Barbosa, J.I. (2014). Optimization of magneto-electro-elastic composite structures using differential evolution, Composite Structures 107: 276-287.

Loja, M.A.R. (2014). On the use of particle swarm optimization to maximize bending stiffness of functionally graded structures, Journal of Symbolic Computation 6162: $12-30$.

Maiz, S., Bambill, D.V., Rossit, C.A. and Laura, P. (2007). Transverse vibration of Bernoulli-Euler beams carrying point masses and taking into account their rotatory inertia: Exact solution, Journal of Sound and Vibration 303(3-5): 895-908.

Majkut, L. (2006). Identification of beams boundary conditions in ill-posed problem, Journal of Theoretical and Applied Mechanics 44(1): 91-105.

Maplesoft (2010). Maple 14.

Martinović, G., Bajer, D. and Zorić, B. (2014). A differential evolution approach to dimensionality reduction for classification needs, International Journal of Applied Mathematics and Computer Science 24(1): 111-122, DOI: 10.2478/amcs-2014-0009.

Mboup, M., Join, C. and Fliess, M. (2009). Numerical differentiation with annihilators in noisy environment, $\mathrm{Nu}$ merical Algorithm 50(4): 439-467.

Meirovitch, L. (2001). Fundamentals of Vibrations, McGraw-Hill, Boston, MA.

Nallim, L.G. and Grossi, R.O. (1999). A general algorithm for the study of the dynamical behaviour of beams, Applied Acoustics 57(4): 345-356.

Posiadała (1997). Free vibrations of uniform Timoshenko beams with attachments, Journal of Sound and Vibration 204(2): 359-369.
Price, K., Storn, R. and Lampinen, J. (2005). Differential Evolution: A Practical Approach to Global Optimization, Natural Computing Series, Springer, New York, NY.

Rasmussen, C.E. and Williams, C.K.I. (2005). Gaussian Processes for Machine Learning, The MIT Press, Cambridge, MA.

Reed, H.M., Nichols, J.M. and Earls, C.J. (2013). A modified differential evolution algorithm for damage identification in submerged shell structures, Mechanical Systems and Signal Processing 39(1-2): 396-408.

Savoia, M. and Vincenzi, L. (2008). Differential evolution algorithm for dynamic structural identification, Journal of Earthquake Engineering 12(5): 800-821.

Silva, T.A.N. and Loja, M.A.R. (2013). Differential evolution on the minimization of thermal residual stresses in functionally graded structures, in A. Madureira, C. Reis and V. Marques (Eds.), Computational Intelligence and Decision Making-Trends and Applications, Springer-Verlag, Dordrecht, pp. 289-299.

Silva, T.A.N. and Maia, N.M.M. (2011). Elastically restrained Bernoulli-Euler beams applied to rotary machinery modelling, Acta Mechanica Sinica 27(1): 56-62.

Silva, T.A.N. and Maia, N.M.M. (2013). Modelling a rotating shaft as an elastically restrained Bernoulli-Euler beam, $E x$ perimental Techniques 37(5): 6-13.

Storn, R. and Price, K. (1997). Differential evolution-a simple and efficient heuristic for global optimization over continuous spaces, Journal of Global Optimization 11(4): 341-359.

Vaz, J.C. (2008). Análise do comportamento dinâmico de uma viga de Euler-Bernoulli escalonada com apoios elasticamente variáveis, Master's thesis, Universidade Federal de Itajubá, Itajubá, MG.

Wang, L. and Yang, Z. (2011). Identification of boundary conditions of tapered beam-like structures using static flexibility measurements, Mechanical Systems and Signal Processing 25(7): 2484-2500.

Wu, J.S. and Chen, D.W. (2003). Bending vibrations of wedge beams with any number of point masses, Journal of Sound and Vibration 262(5): 1073-1090.

Yoneyama, S. and Arikawa, S. (2012). Identification of boundary condition from measured displacements for linear elastic deformation fields, Procedia IUTAM 4: 215-226.

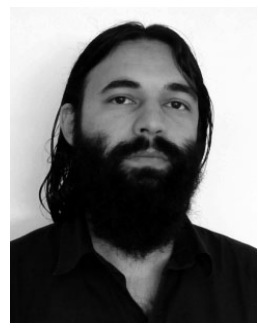

Tiago Alexandre Narciso da Silva is a Ph.D candidate at Instituto Superior Técnico (IST), University of Lisbon, Portugal. He obtained his M.Sc. in mechanical engineering from Lisbon Polytechnic Institute (IPL) in 2009. He has been with Instituto Superiora de Engenharia de Lisboa (ISEL/IPL) as an assistant since 2009. His research work at LAETA, IDMEC Research Institute (IDMEC/IST), is focused on structural model updating, damage detection, optimization. 


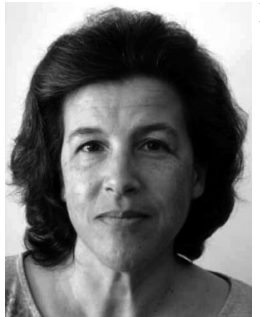

Maria Amélia Ramos Loja is an assistant professor at the Mechanical Engineering Department (ISEL/IPL) and a senior researcher at LAETA, IDMEC/IST. She obtained her M.Sc. and Ph.D. degrees in mechanical engineering from the University of Lisbon. The main topics of her current research at LAETA, IDMEC/IST Research Institute, are related to the modelling and optimization of composite and adaptive structures, as well as the development of algorithms devoted to $3 \mathrm{D}$ surfaces reconstruction

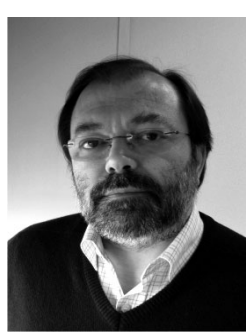

Nuno Manuel Mendes Maia obtained his Master's degree in mechanical engineering from IST (University of Lisbon) and his Ph.D. in mechanical vibrations in 1989 from Imperial College, University of London, UK. He received his habilitation in 2001 from IST. He has authored and co-authored 2 textbooks and more than 160 publications in international journals and conference proceedings. He has participated in and coordinated various research projects in the area of modal analysis and structural vibrations, and has been responsible for the organization of the International Conference on Structural Engineering Dynamics (ICEDyn) since 2002.

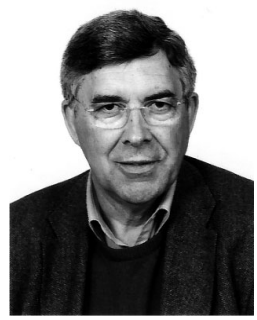

Joaquim Infante Barbosa is a professor at the Mechanical Engineering Department (ISEL/IPL) and a senior researcher at LAETA, IDMEC/IST. $\mathrm{He}$ obtained his M.Sc. and Ph.D. degrees in mechanical engineering in 1984 and 1993, respectively, from the University of Lisbon. He has authored or co-authored over 20 scientific publications in international journals. The main topics of his current research at LAETA, IDMEC/IST Research Institute, are damage of composites and

structural optimization

Received: 11 October 2013

Revised: 31 July 2014

Re-revised: 19 October 2014 